\title{
Large-scale transcriptome sequencing and gene analyses in the crab-eating macaque (Macaca fascicularis) for biomedical research
}

\author{
Jae-Won Huh ${ }^{1,3+}$, Young-Hyun Kim ${ }^{1,3+}$, Sang-Je Park' ${ }^{1,2+}$, Dae-Soo Kim ${ }^{4}$, Sang-Rae Lee ${ }^{1}$, Kyoung-Min Kim ${ }^{1,3}$, \\ Kang-Jin Jung ${ }^{1}$, Ji-Su Kim', Bong-Seok Song ${ }^{1}$, Bo-Woong Sim', Sun-Uk Kim,3, Sang-Hyun Kim ${ }^{1}$ and \\ Kyu-Tae Chang ${ }^{1,3^{*}}$
}

\begin{abstract}
Background: As a human replacement, the crab-eating macaque (Macaca fascicularis) is an invaluable non-human primate model for biomedical research, but the lack of genetic information on this primate has represented a significant obstacle for its broader use.

Results: Here, we sequenced the transcriptome of 16 tissues originated from two individuals of crab-eating macaque (male and female), and identified genes to resolve the main obstacles for understanding the biological response of the crab-eating macaque. From 4 million reads with 1.4 billion base sequences, 31,786 isotigs containing genes similar to those of humans, 12,672 novel isotigs, and 348,160 singletons were identified using the GS FLX sequencing method. Approximately $86 \%$ of human genes were represented among the genes sequenced in this study. Additionally, 175 tissue-specific transcripts were identified, 81 of which were experimentally validated. In total, 4,314 alternative splicing (AS) events were identified and analyzed. Intriguingly, $10.4 \%$ of AS events were associated with transposable element (TE) insertions. Finally, investigation of TE exonization events and evolutionary analysis were conducted, revealing interesting phenomena of human-specific amplified trends in TE exonization events.
\end{abstract}

Conclusions: This report represents the first large-scale transcriptome sequencing and genetic analyses of $M$. fascicularis and could contribute to its utility for biomedical research and basic biology.

\section{Background}

Crab-eating macaques (Macaca fascicularis) are one of the most frequently used and studied species for biomedical research [1]. Due to the broad range of habitats, they have various common names including crab-eating macaque, cynomolgus macaque, Philippine monkey, and long-tailed macaque. Numerous wild crab-eating macaques are distributed in Southeast Asia, including Indonesia, Philippines, Myanmar, Vietnam, and Thailand [2]. They inhabit various habitats including primary, secondary, coastal, mangrove, and riverine forests and areas near villages. Diurnal and

\footnotetext{
* Correspondence: changkt@kribb.re.kr

${ }^{\dagger}$ Equal contributors

${ }^{1}$ National Primate Research Center, Korea Research Institute of Bioscience and Biotechnology (KRIBB), Ochang, Chungbuk 363-883, Republic of Korea ${ }^{3}$ University of Science \& Technology, National Primate Research Center, KRIBB, Chungbuk 363-883, Republic of Korea

Full list of author information is available at the end of the article
}

arboreal crab-eating macaques belong to the infraorder Catarrhini, superfamily Carecopithecoidea, family Cercopithecidae, and genus Macaca.

With the aid of fossil records and comparative DNA sequence analysis, genus macaques and humans have diverged from a common ancestor between 25 and 31 million years ago [3]. This evolutionary relationship has made this primate as a more suitable experimental animal model than rodents, dogs, and pigs and may lead to its widespread use for the translational studies for drug testing [1]. Among the genus Macaca, Rhesus and crab-eating macaque is representative species which were widely used as a non-human primate model for biomedical research. However, the rhesus macaque is the most frequently used primate as a non-human primate model [4]. In the United States, more than $60 \%$ of monkeys housed in National Institutes of Health (NIH)-supported facilities are rhesus macaques [5].

\section{Ciomed Central}


Furthermore, $65 \%$ of the monkeys used for experimental research each year are rhesus macaques. In 2007, first draft genome sequences of rhesus macaque genome was published [4]. These worldwide trends in use and accumulated genome information data may lead to the assumption that the rhesus macaque is the ideal nonhuman primate model. However, the event of "export ban of rhesus monkey from India in 1977" had restricted the usage of Indian subspecies of the rhesus macaque and accelerate the building of self-sustaining breeding colonies in the US. Therefore, researchers who want to have a research with rhesus monkey in the outside of US have some problems, they have concerned the chineseorigin rhesus macaque and crab-eating macaque from south asia [6]. Furthermore, the crab-eating macaque has important advantages, including (1) easy handling derived from a smaller body size ( $\lesssim ~ 412-648 \mathrm{~mm}$, + 385-503 mm vs. त 483-635 mm, o 470-531 mm),

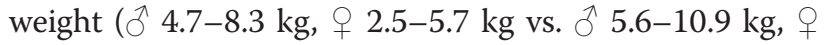
4.4-10.9 kg) and longer tails than rhesus macaques [7]; (2) low cost and easy availability for experimental use; and (3) lack of seasonal fertility, which may affect efficient experiments and scheduling in the large-scale housing of experimental monkeys [8]. Finally, abundant gene information is available for the crab-eating macaque. Greater numbers of EST and full-length cDNA library sequences are available in the NCBI database for crab-eating macaque [9-15]. And recently their draft genome sequences also available in the EBI database $[6,16]$. Therefore, crab-eating macaque could be a excellent experimental primate animal models for biomedical studies.

In an in-depth examination of the published papers from 2010 to 2011 indicated that pharmacology field for safety and toxicity testing of newly developed drugs was the most frequently encountered [17-20]. In particular, the crab-eating macaque was used predominantly in brain research, the neurosciences, and clinical research [21-24]. Furthermore, experimental primate model have been developed by four different ways of simple replacement, induced, infection, and surgical. The induced method involved treatment with specific chemicals (e.g., 1-methyl-4-phenyl-1,2,3,6-tetrahydropyridine (MPTP) or streptozotocin (STZ)[25-27], whereas the surgical method (e.g., middle cerebral artery occlusion model for ischemia) were created through specific types of surgery [28]. The infection method was simpler than previously described since humans and the crab-eating macaque have numerous "anthroponosis" (the opposite of "zoonosis"), including influenza, tuberculosis, and hepatitis [29]. Lastly, simple replacement method was the usage of natural crab-eating monkey for specific purpose (e.g., drug safety or efficacy testing) [30].

From now, numerous disease models, including aging, alcohol abuse, Alzheimer's disease, amenorrhea, asthma, diabetes, epilepsy, menopause, obesity, osteoporosis, Parkinson's disease, plague, variola, vascular disease, and various infection disease models, have been developed and used [31-46]. However, small amount of transcript sequences of crab-eating macaque could be a weak point to be a good experimental animals for biomedical application. If we have abundant transcript sequences for crab-eating macaque, we could design the whole gene probe sequences for microarray analyses. And also, due to the insufficient transcript sequences, we could not analyze the alternatively spliced transcripts in different tissues. Recent accumulated transcriptome information underlined that AS event is an important molecular mechanism since it can generate different functional units for transcriptome and proteome diversity using limited genetic sources [47-49]. And also human transcriptome studies with different human tissues show different AS patterns derived by tissue-specific alternative promoters and polyadenylation [50-52]. However, sometimes aberrant changes in alternative splicing could occur the human disease (e.g. retinitis pigmentosa or cystic fibrosis) [53,54]. And A few number of papers have reviewed the association between alternative splicing and disease [55-58]. Among the different AS mechanisms, TE exonization is intriguing AS events [59]. Specifically, small amount of TEs show the tissue specific and species specific characters [60]. That means that TE exonization event could be a one of the important AS events. Therefore, AS is not a simple molecular aspect of RNA transcription, rather it represents a highly controlled and evolved molecular mechanism for generating genetic diversity using limited DNA resources. And also AS control mechanisms are major growing topics in biomedical researches. Hence, the investigation of the AS events in specific genes is another means of novel gene or disease gene identification and characterization steps. However, these kinds of applications with crab-eating macaque for advanced biomedical research could be achieved by the massive amount of transcript sequences and information.

In this study, we carried out a whole-transcriptome sequencing analysis of 16 tissues from Macaca fascicularis using GS FLX sequencing to generate massive transcript information for the improvement of biomedical use. More than 4 million raw reads were created and assembled, resulting in 35,524 isogroups, 44,458 isotigs, 54,858 contigs, and 348,160 singletons. Additionally, we identified and experimentally validated differentially expressed gene (DEG) transcripts. Finally, using the numerous transcript sequences, we analyze the AS and TE events of crab-eating macaque.

\section{Results and discussion}

\section{GS FLX sequencing and gene annotation}

Among the different next generation sequencing methods, we selected the GS FLX sequencing platform. 
Although this platform demanded the high cost for sequencing, longer read length of output sequences are more adequate for the de novo assembly for crab-eating macaque genes $[61,62]$. A total of 4,058,656 raw reads were obtained from the 16 different tissue libraries, with a mean sequenced size length of 355 bp (Additional file 1: Table S1, Additional file 1: Table S2, Additional file 1: Table S3). For rapid assembly and exact gene annotation, all raw reads were divided into 2 groups, clustered reads and unclustered reads, by the clustering method of the BLASTN program with human reference RNA, generating $3,240,337$ reads clustered with human reference RNA, and 818,319 unclustered reads (Additional file 2: Figure S1). Each group was analyzed by GS de novo Assembler v.2.5.3 (Newbler, 454 Life Science). In the clustered group, 38,750 assembled contigs, 31,786 isotigs, and 24,884 isogroups and 99,283 unassembled singletons were generated. However, 132,121 reads were discarded due to excessively short, chimeric, or repetitive sequences. For the clustered isotigs, half of the sequences were larger than 900 bp, and more than 2,400 were longer than 3,000 bp (Additional file 2: Figure S2). Total annotated sequences covered $\sim 86 \%(39,439$ genes $)$ of the human reference genes (Figure 1; Additional file 1: Tables S4-S5). By contrast, 55\% of the sequences (5,915 isotigs and 209,598 singletons) did not match any of the human reference genes (Additional file 2: Figure S1). Although more detailed experimental validations must be performed, these sequences (5,915 isotigs and 209,598 singletons) may be macaque-specific genes that define differences between humans and crab-eating macaques.
Application for OMIM database and KEGG pathway database We then applied our results to the OMIM database (http://www.ncbi.nlm.nih.gov/omim/), which provides information on disorder-related genes that have been functionally well-characterized, and the KEGG pathway database (http://www.genome.jp/kegg/pathway.html), a representative molecular pathway database specifically for disease-related pathways. In the OMIM database, we collected all of the available gene sets for calculation of coverage. Of the 2,579 disorder-related genes in the OMIM database (Additional file 1: Table S6), 1,935 genes $(75.02 \%)$ were covered by our results (Additional file 1: Table S7), indicating that the gene information from our sequencing could lead to an enhanced understanding of the genetic responses to specific experimental conditions in disease-related research on the crabeating macaque.

MPTP treatment of the crab-eating macaque is one of the most well established models of Parkinson's disease [39]. Therefore, we applied our results to the investigation of Parkinson's disease (map05012) in the KEGG pathway. In general, first step of disease mechanism research is the identification of full-length gene sequences, specifically coding sequences (CDS), using cDNA library or RACE experiments for the investigation of a specific disease. Then other following steps of in vitro or in vivo experiments are applied for the characterization of specific disease. Therefore, the identification of intact CDS in genes was our primary goal. In the KEGG pathway database, 129 Parkinson's disease genes were registered. We manually tested the existence of open-reading frame sequences and compared the existence of full-length CDS

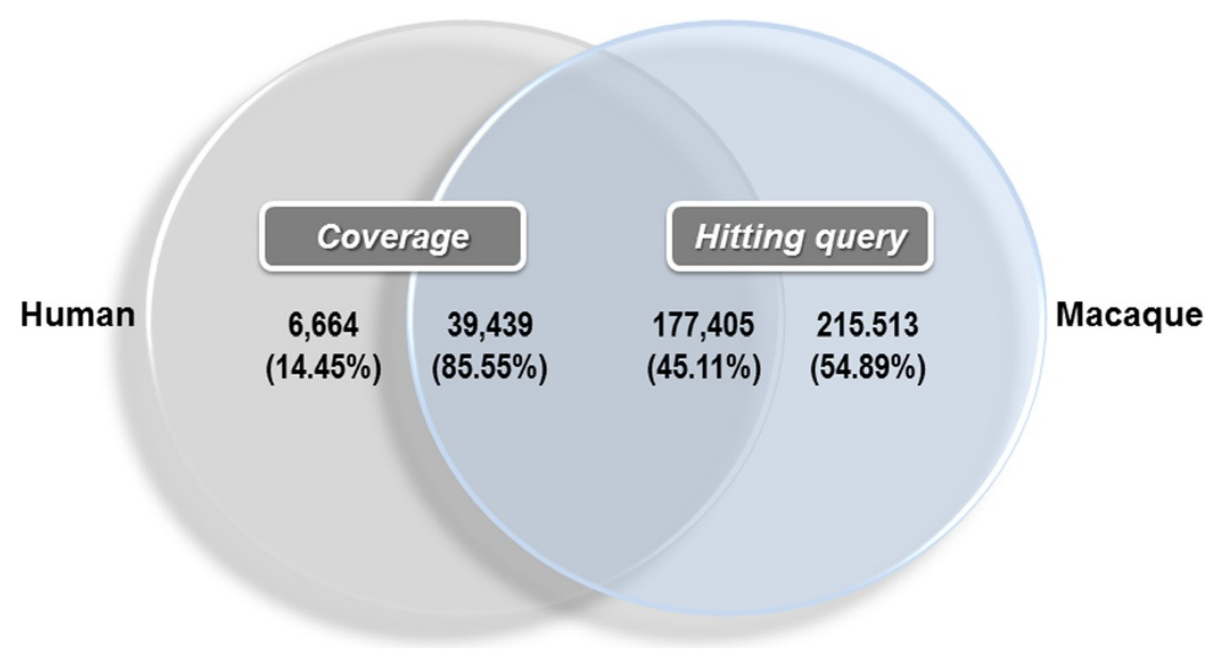

Figure 1 Comparative analysis of crab-eating macaque transcriptome sequences with human reference genes. Human reference gene coverage was calculated using the BLASTX program. A total of 177,405 crab-eating macaque transcripts (45.11\%) were matched to 39,439 human reference RNA sequences (85.55\%). 
with our sequencing data (data not shown). Our results indicated that total 115 genes (89\%) harbor the intact fulllength CDS (101 genes) or truncated CDS or UTR sequences (14 genes). These high rate of identification of intact full-length sequences are coincided the property of GS FLX sequencing platform (long-read sequencing) $[61,62]$. Although, we did not validated the other diseaserelated genes in OMIM database, our results can clearly reduce the cost and experimental efforts for the identification of specific disorder-related genes for biomedical research.

\section{Differentially expressed gene analysis and experimental validation}

More than 4 million reads harboring tissue information were used in the assembly steps (Figure 2). Therefore, it was possible to use tissue information to identify differentially expressed genes (DEGs) candidates. Strict filtering conditions were applied for the identification of DEG candidates (more than 100 reads and the use of contigs exclusively expressed in specific tissues). In total, 175 genes were identified as DEG candidates (Additional file 1: Tables S8-S20). Testis (45 genes) and liver (42 genes) showed the largest number of DEG candidates (Table 1). By contrast, the ovary, spleen, cerebrum, and cerebellum did not harbor tissue-specific transcripts. However, when we pooled the cerebrum and cerebellum tissue as brain tissue, one gene, CBLN1 was identified as a DEG candidates.

Identified DEG candidates were subdivided into 3 groups: functionally well-characterized genes in specific tissues, functionally well characterized genes with tissue relatedness not investigated, and functionally not characterized genes with tissue relatedness not investigated. For example, among the 45 testis DEGs, genes including COX6B2, DPY19L2, IZUMO4, PRM2, TSSK6, and H1FNT have been previously investigated as testisspecific transcripts or spermatogenesis-related genes (http://www.ncbi.nlm.nih.gov/gene/). Other genes such as C6orf225, C20orf107, FUNDC2, and LELP1 have not been functionally investigated in any other tissues in previous research, while the CETN1 gene has a specific function in centrosome positioning and segregation [63] but has not been investigated with respect to tissue relatedness. Therefore, these DEGs could be utilized as major target genes for tissue specific transcripts for tissue specific function and novel gene identification in specific tissues. For the experimental validation of DEG candidates, 81 genes were randomly selected and experimentally confirmed by RT-PCR amplification and sequencing procedures (Table 1; Figure 3). Remarkably, more than $95 \%$ of the genes were validated as real DEGs with distinct expression in expected tissues. These

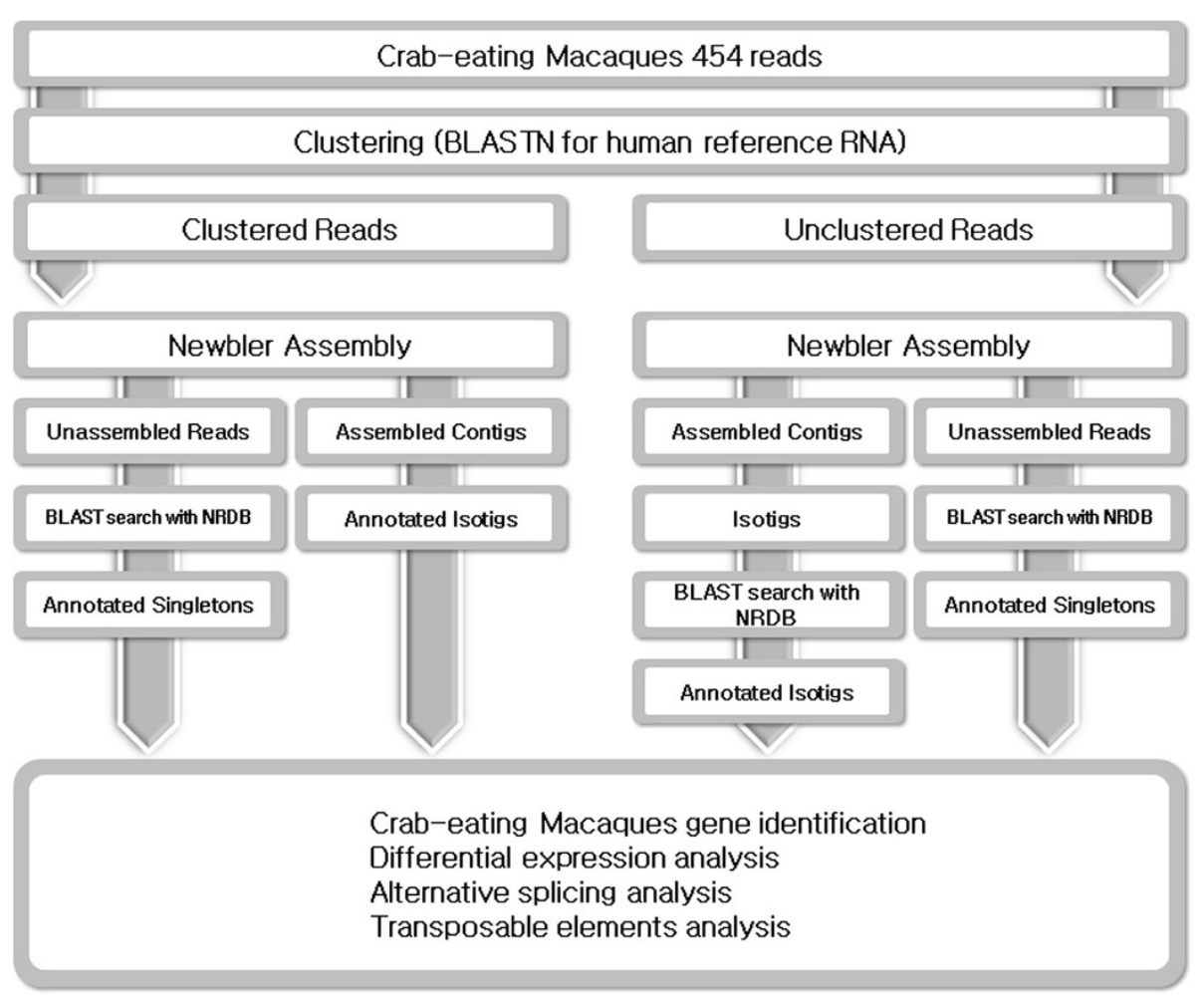

Figure 2 Flow chart for data analysis of the crab-eating macaque. 
Table 1 Identification and validation of tissue-specific transcripts

\begin{tabular}{|c|c|c|c|}
\hline Tissue & $\begin{array}{c}\text { DEG } \\
\text { candidates }\end{array}$ & $\begin{array}{l}\text { Selected DEGs } \\
\text { for experimental } \\
\text { validation }\end{array}$ & Gene Name ${ }^{*}$ \\
\hline Cecum & 4 & 3 & $S L C 12 A 2^{1}, C A 1^{2}, C L C A 4^{3}$ \\
\hline Cerebellum & 1 & 1 & $C B L N 1^{4}$ \\
\hline \multicolumn{4}{|l|}{ Cerebrum } \\
\hline Heart & 3 & 2 & $M Y B P C 3^{5}, \angle D B D 3^{6}$ \\
\hline Kidney & 11 & 10 & 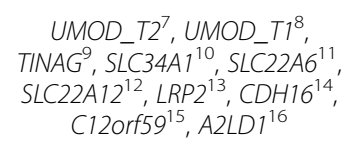 \\
\hline Liver & 42 & 10 & $\begin{array}{c}C Y P 2 B 6^{17}, C 9^{18}, F 9^{19}, T A T^{20} \\
F 13 B^{21}, C R P^{22}, C 8 B^{23}, F G G^{24} \\
G C^{25}, M B L 2^{26^{\prime}}\end{array}$ \\
\hline Lung & 5 & 4 & $\begin{array}{c}\text { SFTPD }^{27}, \operatorname{SFTPB}^{28} \text { SFTPC }^{30} \\
\text { SFTPA }^{29}\end{array}$ \\
\hline Ovary $^{\dagger}$ & 0 & 0 & \\
\hline Pancreas & 22 & 11 & 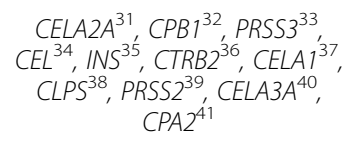 \\
\hline Prostate & 3 & 2 & SEMG $2^{42}, M S M B^{43}$ \\
\hline $\begin{array}{l}\text { Salivary } \\
\text { gland }\end{array}$ & 19 & 11 & 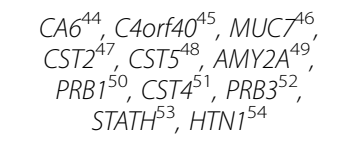 \\
\hline $\begin{array}{l}\text { Skeletal } \\
\text { muscle }\end{array}$ & 11 & 8 & $\begin{array}{c}\text { MYH }^{55}, \text { AMPD } 1^{56}, \text { TPM }^{57} \\
\text { ATP2A } 7^{58}, \text { MYOT }^{59}, \text { MYBPC1 }^{60} \\
\text { MYL1 }\end{array}$ \\
\hline $\begin{array}{l}\text { Small } \\
\text { intestine }\end{array}$ & 2 & 2 & $F A B P 2^{63}, D E F A 6^{64}$ \\
\hline Spleen & 0 & 0 & \\
\hline Stomach & 7 & 5 & $\begin{array}{c}C H I A^{65}, L I P F^{66}, G K N 2^{67}, G K N 7^{68}, \\
P G A 5^{69}\end{array}$ \\
\hline Testis & 45 & 12 & 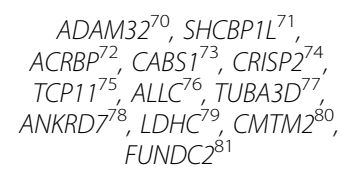 \\
\hline
\end{tabular}

*The superscript numbers (1-81) correspond to the validated gene numbers in Figure 3

+ Ovary samples were not used for experimental validation for the experimental efficiency.

results support the reliability of our sequencing and emphasize the importance of tissue sample preparation when conducting high-throughput sequencing.

\section{Alternative splicing (AS) analysis}

A total of 6,931 manually corrected AS events were identified in the 24,884 clustered isogroups (Additional file 1: Table S21). Total 4,314 isogroup harbored the more than one alternatively spliced transcripts. The average number of AS events was 1.60, and the highest number observed was 63 AS events in the $A K R 1 B 10$ gene (Additional file 1: Table S22). Intriguingly, the human $A K R 1 B 10$ gene shows only one reference mRNA sequence, while the EBI database of Alternative Splicing and Transcript Diversity 1.1 indicated only 5 alternative transcripts for this gene in humans (http://www.ebi.ac.uk/asd/index.html). A careful analysis indicated that AS events occurred more frequently in the $5^{\prime}$ and $3^{\prime}$ regions (2,270 and 2,313, respectively) than the internal regions $(1,727)$ (Additional file 1: Table S22). Further, 274 AS events (10.4\%) were TE related. As a result, $\sim 17 \%$ of the crab-eating macaque isogroups were shown to have alternatively spliced transcripts. This lower rate of AS events in the crab-eating macaque may be explained by 2 alternative interpretations. One is the shortage of total amount of transcript sequences. In the case of human studies, earlier researches indicated that approximately $40 \%-70 \%$ of genes have alternative transcripts. However, advanced high-throughput sequencing and bioinformatic tools have shown that $92 \%-95 \%$ of human genes undergo AS [50,51,64-66]. In addition, different human tissues show different AS patterns because of tissue-specific alternative promoters and polyadenylation [50-52]. Therefore, larger amount of transcript sequences and more diverse tissues or cell types could enhance the AS information. Another is explained by simple lineage specific characters. Because, we already observed the differential alternative splicing between human and chimpanzees [63,67]. And, as indicated in the genome project of chimpanzee and orangutan, different amplification rate and lineage specific of transposable elements could cause the different TE-derive alternative splicing $[68,69]$.

\section{Transposable element (TE) analysis}

Recent growing genomic evidence has indicated that TEs are a valuable genetic resource for transcriptome and proteome diversity [70-73]. Exonization events are one of the AS mechanisms that can occur as a result of TEs, including human endogenous retroviruses (HERVs), short interspersed elements (SINEs), and long interspersed elements (LINEs). Alu (a primate-specific SINE) and LINEs have potential $5^{\prime}$ and $3^{\prime}$ splicing sites for exonization events. Moreover, HERVs and LINEs harbor internal promoters that can control the tissue-specific expression of a gene [59].

Among the different TEs, Alu is the most frequently exonized element. However, in our comparative analysis with human, slight differences in the patterns of $A l u$ exonization were observed. Alu elements underwent an exonization event in $2.38 \%$ of human genes and in $1.76 \%$ of crab-eating macaque genes. Therefore, we extended our analysis to all TEs in human, chimpanzee, crab-eating macaque, rhesus macaque, and marmoset monkey for the comparative analysis of primates. Intriguingly, this extended study indicated a increase pattern in TE composition over primate evolution and different TE-exonization events between rhesus macaque and cran-eating macaque (Figure 4). 


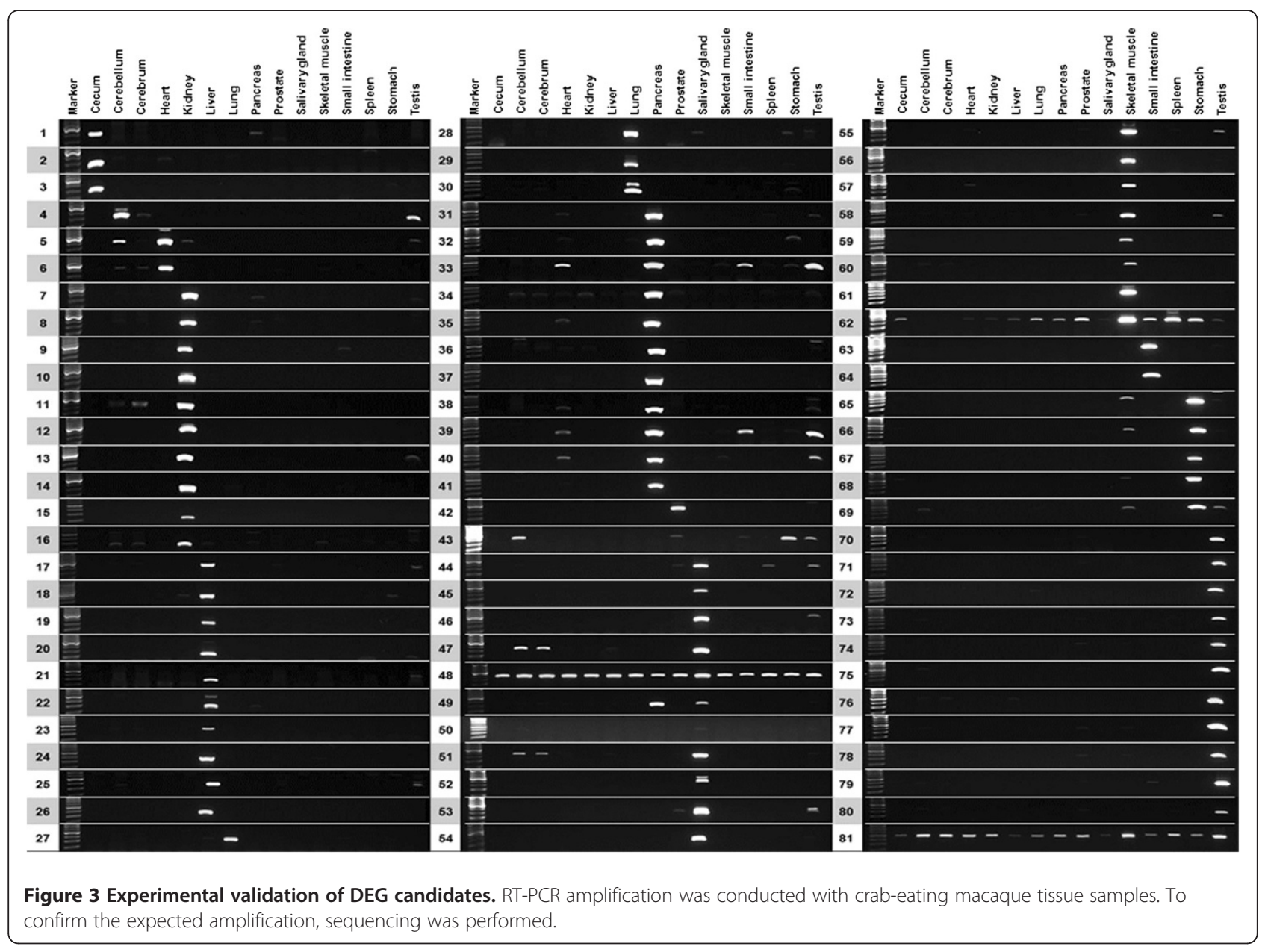

Although primate gene information was not sufficient to conclude from our results that amplified TE composition is a human-specific event, our results do indicate that TE exonization events were amplified over primate evolution and notably in humans. These types of amplified TE exonization events in humans could enhance the transcriptome and proteome diversity with fixed genome sequences in comparison with non-human primates. However, we also explained the results of Figure 4 as decrease pattern in TE composition. Because the probability is very low, recent studies newly raised the $A l u$ recombination-mediated deletion (ARMD) and L1 recombination-associated deletions (LRMD) mechanisms which could remove the internal sequences by homologous recombination of "Alu" or "LINE" elements [74,75]. In the case of rhesus macaque and marmoset, the results of low-level TE-exonization rate in comparison with other species are seems to be occurred by the lack of transcript sequences (http://www.ncbi.nlm. nih.gov/Taxonomy/Browser/). Because most of reference mRNA sequences are identified by computational screening without the intensive support of numerous EST or cDNA sequences.

\section{Broad range of utility of crab-eating macaque gene information}

The results of our study have implications for various fields of research. First, the massive number of transcriptome sequences (approximately 4 million sequences in 16 tissues) could be used as a draft of the crab-eating macaque gene sequences. In addition, the modified and combined gene information could be used for the production of DNA probe sequences for microarray analysis. Specifically, the company Agilent provides a customized probe design service using industrial-scale inkjet technology (http://www.genomics.agilent.com/). Therefore, crab-eating macaque microarray chips could be designed for specific experiments and more rapid and accurate gene expression profiling is possible in a single experiment. For example, to investigate specific drugs for Parkinson's disease, customized microarray chips harboring the 129 Parkinson's disease-related crab-eating macaque genes from the KEGG pathway database could be prepared.

Second, crab-eating macaque gene information coupled with gene information from the rhesus macaque could be used to resolve the mystery of speciation events 


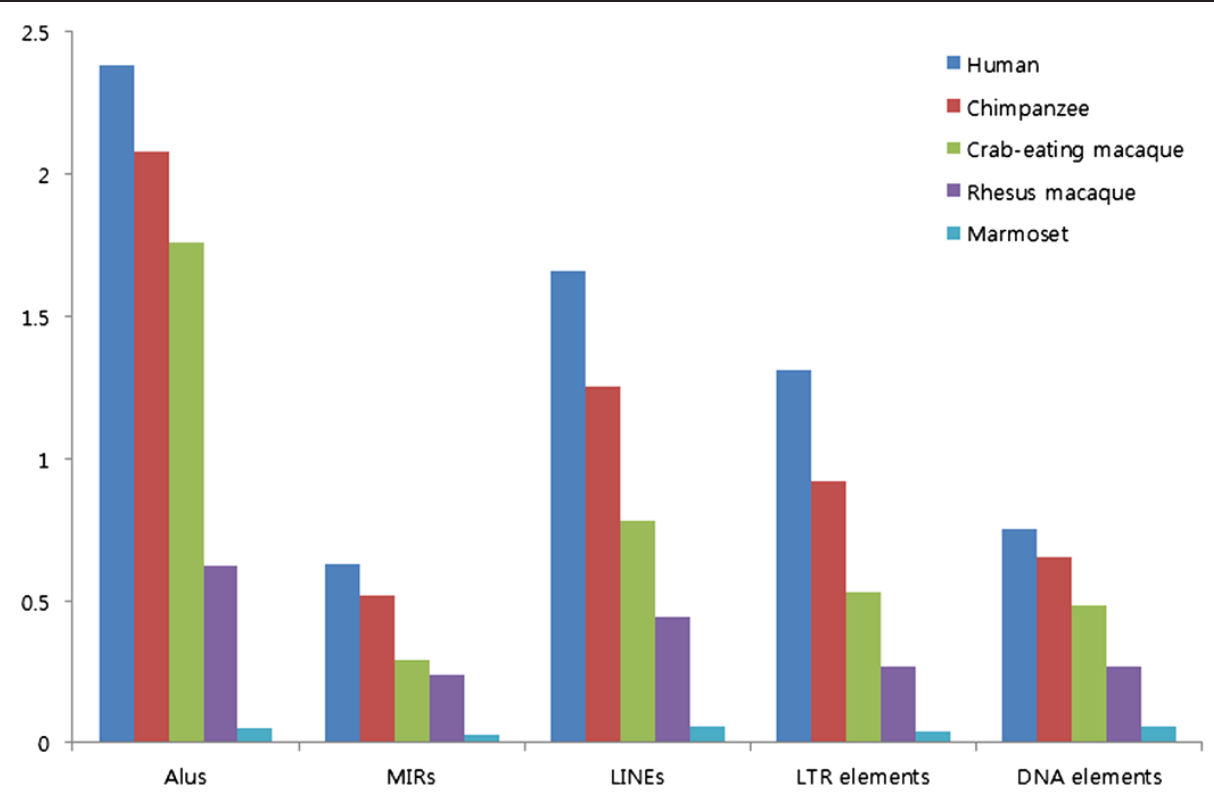

Figure 4 Comparative analysis of transposable element exonization events in primates. Human, chimpanzee, crab-eating macaque, rhesus macaque, and marmoset monkey gene information were used for our analysis.

between closely related species. The average genetic divergence between crab-eating macaque and rhesus macaque is $0.4 \%-0.5 \%$, and their evolutionary relationship is closer than that between human and chimpanzee [14]. Therefore, large-scale transcript sequences could help to trace the evolutionary root of the speciation event. Third, gene sequencing of the crab-eating macaque could accelerate the completion of a genome project for this primate. Recently draft genome sequences also available (http://www.ebi.ac.uk/asd/index.html). Hence, reanalysis and diverse application could be possible for the analysis of genome and transcripme in crab-eating macaque. Fourth, the $175 \mathrm{DEGs,}$ including the 81 experimentally validated DEGs, represent candidate genes with tissue-specific functions. Specifically, two of gene groups of functionally well characterized genes with tissue relatedness not investigated, and functionally not characterized genes with tissue relatedness not investigated, could be a valuable sources for tissue specific functional study and novel function analysis in specific tissue, respectively. Fifth, the AS and TE exonization analysis could be used for comparative analysis of crab-eating macaque with other species. Although, the data set are not sufficient for other application, our results are to be used as basic information to understand the transcriptome of crab-eating macaque. Finally, our open data base are very useful for numerous researchers who are interested in the gene information of crab-eating macaque, specifically unskilled researchers in genomics and bioinformatics technique.

\section{Conclusions}

We sequenced the transcriptome of 16 different tissues from M. fascicularis for the biomedical usage. We found that $\sim 86 \%$ of human genes are represented in the ones sequenced in this study. Therefore our results of gene information could be used for understanding the biological response of the crab-eating macaque for safety and efficacy testing. Additionally, 175 tissue-specific genes were identified, with 81 of them experimentally validated. We identified and analyzed 4,314 alternative splicing (AS) events and positive selected genes. Intriguingly, $10.4 \%$ of the AS events were associated with transposable element (TE) insertions. And human-specific amplified trends of TE exonization event are also revealed during the primate evolution. Our research is the first large-scale transcriptome sequencing and gene analyses. Therefore, this result could be valuable genetic resources for biomedical research and improve our understanding of primate evolution.

\section{Methods}

Specific pathogen free (SPF) crab-eating macaques

Adult male (5 years old) and female (6 years old) crabeating macaques (Macaca fascicularis) weighing between $4 \mathrm{~kg}$ and $7 \mathrm{~kg}$ were used. Their origin is vietnam. All animals were provided by the National Primate Research Center (NPRC) of Korea. In our experiments, specific pathogen free (SPF) animals were used. All animals underwent a complete physical, viral, bacterial, and parasite examination. On physical examination, SPF animals were 
examined for criteria, including coat condition, appearance, weight, sex, and date of birth. Enzyme immunoassay was performed to detect viruses such as BV; STLV-1 and -2; SIV; SRV-1, -2, and -5; and SVV. In addition, tests were performed to detect Mycobacterium tuberculosis (TB), Shigella spp., Salmonella spp., and Yersinia spp. For the TB skin test, all animals were tested by an intradermal injection in the eyelid, and the remaining bacterial examination items were checked by fecal culture tests. In our SPF animals, all items in the above tests were negative.

\section{Sample preparation for GS FLX sequencing and gene annotation}

The most important issue for transcriptome sequencing is the preparation of fresh and healthy tissue samples. Therefore, specific pathogen free (SPF) one male and one female adult crab-eating macaques were selected. Additionally, perfusion with diethylpyrocarbonate (DEPC)treated phosphate buffered saline (PBS) was conducted via the common carotid artery with RNase inhibitors to inhibit blood contamination and promote recovery of intact RNA molecules from the tissue samples. Sixteen tissue samples were collected from one male and one female crab-eating monkeys (1. Cecum, 2. Cerebellum, 3. Heart, 4. Kidney, 5. Liver, 6. Lung, 7. Ovary, 8. Pancreas, 9. Prostate, 10. Salivary gland, 11. Skeletal muscle, 12. Small intestine, 13. Spleen, 14. Stomach, 15. Testis, and 16. Cerebrum).

\section{Ethics statement}

All animal procedures and study design were conducted in accordance with the Guidelines of the Institutional Animal Care and Use Committee (KRIBB-AEC-11010) in Korea Research Institute of Bioscience and Biotechnology (KRIBB).

\section{RNA isolation and mRNA subtraction}

Total RNA was extracted from 16 different crab-eating monkey tissues using the Trizol reagent (Invitrogen), and total RNAs were validated by RNA electrophoresis in agarose gels containing formaldehyde. Two distinct ribosomal RNA bands (28 S and $18 \mathrm{~S}$ ) were confirmed. Pure mRNA was subtracted using the PolyA Tract mRNA isolation system (Promega).

\section{CDNA synthesis and poly $(A)$ tail removal}

First strand cDNA synthesis was conducted using the RevertAid H Minus First Strand cDNA Synthesis Kit (Fermentas) using oligo(dT) primers optimized for the 454 sequencing procedures (5'- GAGCTAGTTCTG GAG $\left.(\mathrm{T})_{16} \mathrm{VN}-3^{\prime}\right)$. Second strand cDNA was synthesized by DNA pol I and RNase $\mathrm{H}$ (Fermentas), and the poly (A) tail was removed using a specific enzyme (Gsul).

\section{Library preparation for GS FLX sequencing}

The first step of library preparation involves the fragmentation of the high molecular weight DNA sample into smaller molecular species appropriate for sequencing using GS FLX Titanium chemistry. This fragmentation is performed by nebulization, which shears doublestranded DNA into fragments ranging from about 400 to 1000 base pairs. This population of smaller-sized DNA species, generated from a single DNA sample, is referred to as a "library." Approximately 3-5 $\mu \mathrm{g}$ cDNA was used to generate the DNA library for Genome Sequencer FLX Titanium (Roche, Mannheim, GE). The fragment ends were polished (blunted), and 2 short adapters were ligated onto both ends. The adapters provide priming sequences for both amplification and sequencing of the sample library fragments, as well as the "sequencing key", a short sequence of 4 nucleotides used by the system software for base calling and, following repair of any nicks in the double-stranded library, release of the unbound strand of each fragment (with 5'Adaptor A). Finally the quality of the library of singlestranded template DNA fragments (sst DNA library) was assessed using a 2100 BioAnalyzer (Agilent, Waldbronn, GE), and the library was quantified, including a functional quantification to determine the optimal amount of the library to use as input for emulsion-based clonal amplification.

\section{Emulsion PCR}

Single "effective" copies of template species from the DNA library to be sequenced were hybridized to DNA Capture Beads. The immobilized library was then resuspended in the amplification solution, and the mixture was emulsified, followed by PCR amplification. After amplification, the DNA-carrying beads were recovered from the emulsion and enriched. The second strands of the amplification products were melted away as part of the enrichment process, leaving the amplified singlestranded DNA library bound to the beads. The sequencing primer was then annealed to the immobilized amplified DNA templates.

\section{Sequencing}

After amplification, the DNA-carrying beads were set into the wells of five and a half PicoTiterPlate device (PTP) such that each well contained a single DNA bead. The loaded PTP was then inserted into the Genome Sequencer FLX instrument, and sequencing reagents were sequentially flowed over the plate. Information from all the wells of the PTP is captured simultaneously by a camera and can be processed in real time by the onboard computer. The sequencing procedure was conducted on a Genome Sequencer FLX Titanium instrument (Roche, Mannheim, GE) at Macrogen in Korea. 


\section{Sequence assembly and gene annotation}

A total of 4,058,656 raw reads obtained from the 16 libraries were used for our analysis. For rapid assembly and exact gene annotation, all raw reads into were divided into 2 groups, clustered reads and unclustered reads, by the clustering method of the BLASTN program with human reference RNA (Additional file 2: Figure S2). This method generated 3,240,337 reads clustered with human reference RNA and 818,319 unclustered reads. Each group was analyzed by GS de novo Assembler v.2.5.3 (Newbler, 454 Life Science). The clustered group generated 38,750 assembled contigs, 31,786 isotigs, and 24,884 isogroups and 99,283 unassembled singletons. However, 132,121 reads were discarded due to short, chimeric, or repetitive sequences. The unclustered group generated 16,108 assembled contigs 12,672 isotigs, and 10,640 isogroups and 248,877 unassembled singletons. In addition, 57,613 reads were also discarded.

Two different gene annotation strategies were conducted in the clustered and unclustered groups. In the clustered group, initial gene information obtained by clustering with human reference RNA was used for the gene annotation. However, in the case of the unclustered group and unassembled singleton sequences, the BLASTX program was used with the nr70 database. The CD-HIT program (http://www.bioinformatics.org/ cd-hit/) was used to build the nr70 database. If gene annotation was conducted, Gene Ontology (GO) searching (http://www.geneontology.org/) and Kyoto Encyclopedia of Genes and Genomes (KEGG) analysis (http://www. genome.jp/kegg/) were performed.

\section{KEGG pathway analyses}

By overlaying expression data onto biological pathways, established and novel relationships among genes can be explored. These pathways give key information about the functional and metabolic organization of cellular and biological systems within organisms. Therefore, putative crabeating macaque genes incorporate KEGG pathway information. The pathway analysis pipeline extracts EC numbers from the descriptions of UniProt results, and these EC numbers are mapped with KEGG pathway information.

\section{Coverage calculation}

Using the annotated gene information, our sequences were compared with human unigene and reference sequences. Our sequences were analyzed using the BLASTN program with an expectation value of $1 \mathrm{e}^{-20}$. If one match occurred between human and crab-eating macaque sequences, the one match was interpreted as a covered result. Additionally, Online Mendelian Inheritance in Man (OMIM) gene sets were applied for disease-related gene research (http://www.ncbi.nlm.nih.gov/omim).

\section{Differentially expressed gene (DEG) analysis}

Sixteen different tissue samples were collected and sequenced. Thus, over 4 million reads harboring different tissue information were available for the DEG analysis. DEG information was extracted by counting the read information. Exclusively tissue-specific contigs (only allowed $100 \%$ ) that contained a minimum of 100 reads were selected. For the experimental validation, 81 randomly selected DEGs were validated.

\section{Reverse transcriptase polymerase chain reaction (RT-PCR) amplification and sequencing procedure}

Locus-specific primer pairs were used for the RT-PCR amplification of 81 DEGs (Additional file 1: Table S23). If possible, 2 distant exons were used for constructing primer pairs to reduce non-specific PCR bands resulting from genomic contamination. In the validation steps, 15 tissues samples are used for the experimental efficiency (We removed the ovary samples). M-MLV reverse transcriptase with an annealing temperature of $42^{\circ} \mathrm{C}$ was used for the reverse transcription reaction with an RNase inhibitor (Promega). Control PCR amplification was also performed on pure mRNA samples that were not subjected to reverse transcription, indicating that the prepared mRNA samples did not contain genomic DNA. RT-PCRs were carried out for 30 cycles at specific annealing temperatures. To validate amplified products, RT-PCR products were separated on a $1.5 \%$ agarose gel, purified using a gel extraction kit (GeneAll), and cloned into the pGEM-T-easy vector (Promega). The cloned DNA was isolated using a plasmid DNA mini-prep kit (GeneAll). Sequencing was conducted by a commercial sequencing company (Macrogen).

\section{Transposable element (TE) analysis}

The TEs included in the human reference RNAs, chimpanzee reference RNAs, rhesus reference RNAs, marmoset reference RNAs, and clustered assembly contigs were analyzed for comparative TE analysis. The TEs were identified by the RepeatMasker program (http:// repeatmasker.genome.washington.edu) with various repeat sequences from the Repbase Update.

\section{Alternative splicing (AS) analysis}

For the AS analysis, the Newbler2.5 assembly result files (454AllContig.fna, 454Isotigs.fna, and 454IsotigsLayout. txt) were modified. Among these result files, the 454IsotigsLayour.txt file demonstrated the relationships between isotigs and contigs in specific isogroups. Therefore, the alternatively spliced isogroup information was collected. Among the AS data, only clustered and annotated isogroups were analyzed for the comparative analysis with humans. However, in the case of crab-eating macaque, detailed phenomena could not be investigated 
because no crab-eating macaque genome sequences are available. For a detailed analysis, the AS data was analyzed manually. The $5^{\prime}$ and $3^{\prime}$ alternative exon and internal exon units that could occur by exon creation or loss (Additional file 1: Table S22) and the TE-related AS were counted. In the manual analysis, specific exons harboring a TE in the marginal regions of exons were designated as TE-related AS (Additional file 2: Figure S3).

\section{Additional files}

Additional file 1: Table S1. The information of GS FLX sequencing procedure. Table S2. The summary of sequencing procedure in 16 different tissues. Table S3. The summary of Crab-eating Macaques 454 sequencing. Table S4. Coverage calculation of crab-eating macaque through human unigene and human reference gene. Table S5. Calculation of hitting query of crab-eating macaque with human unigene and human reference. Table S6. The list of Gens used for OMIM analysis. Table S7. The list of OMIM genes covered by crab-eating macaque. Table S8. The list of DEG candidate in Brain. Table S9. The list of DEG candidate in Cecum. Table S10. The list of DEG candidate in Heart. Table S11. The list of DEG candidate in Kidney. Table S12. The list of DEG candidate in Liver. Table S13. The list of DEG candidate in Lung. Table S14. The list of DEG candidate in Pancreas. Table S15. The list of DEG candidate in Prostate. Table S16. The list of DEG candidate in Salivary gland. Table S17. The list of DEG candidate in Skeletal muscle. Table S18. The list of DEG candidate in Small intestine. Table S19. The list of DEG candidate in Stomach. Table S20. The list of DEG candidate in Testis. Table S21. Summary of alternative splicing events in crabeating macaque. Table S22. Manually analyzed results of alternative splicing in crab-eating macaque. Table S23. Primer infromation for DEG validation.

Additional file 2: Figure S1. Flowchart for bioinformatic analysis. Figure S2. Length distribution of crab-eating macaque isotigs. For the analysis of length distribution, clustered and unclustered isotigs were analyzed. Figure S3. Manual selection method for TE-derived AS events.

\section{Abbreviations}

AS: Alternative Splicing; BP: Biological Process; CC: Cellular Component; CDS: Coding Sequences; DEG: Differentially Expressed Gene;

DEPC: Diethylpyrocarbonate; GO: Gene Ontology; HERVs: Human Endogenous Retroviruses; KEGG: Kyoto Encyclopedia of Genes and Genomes; LINEs: Long Interspersed Elements; MPTP: 1-methyl-4-phenyl-1,2,3,6tetrahydropyridine; NIH: National Institutes of Health; NPRC: National Primate Research Center; OMIM: Online Mendelian Inheritance in Man; PBS: Phosphate Buffered Saline; PTP: PicoTiterPlate; RT-PCR: Reverse Transcriptase Polymerase Chain Reaction; SINEs: Short Interspersed Elements; SPF: Specific Pathogen Free; TB: Tuberculosis; TE: Transposable Element.

\section{Competing interests}

Authors declare that they have no competing interests.

\section{Accession numbers and database}

The data have been deposited in the DDBJ under accession number DRA000436. The assembled sequences are also freely available from http:// 203.239.28.13/macaca/

\section{Authors' contributions}

KTC managed the project. JWH analyzed the sequencing data. JWH, YHK and SJP wrote the manuscript. DSK conducted the bioinformatic analysis. KMK, KJJ and SRL conducted the housing and sampling the crab-eating macaques. YHK, SJP, BSS, JSK, BWS, SUK and SHK validated the sequencing data. All authors read and approved the final manuscript.

\section{Acknowledgements}

This research was supported by a grant from the KRIBB Research Initiative Program (KGM4241231).

\section{Author details}

${ }^{1}$ National Primate Research Center, Korea Research Institute of Bioscience and Biotechnology (KRIBB), Ochang, Chungbuk 363-883, Republic of Korea. ${ }^{2}$ Department of Biological Sciences, College of Natural Sciences, Pusan National University, Busan 609-735, Republic of Korea. ${ }^{3}$ University of Science \& Technology, National Primate Research Center, KRIBB, Chungbuk 363-883, Republic of Korea. ${ }^{4}$ Genome Resource Center, Korea Research Institute of Bioscience and Biotechnology (KRIBB), Daejeon 305-806, Republic of Korea.

Received: 10 January 2012 Accepted: 13 April 2012

Published: 4 May 2012

\section{References}

1. Carlsson HE, Schapiro SJ, Farah I, Hau J: Use of primates in research: a global overview. Am J Primatol 2004, 63:225-237.

2. Fooden J: Systematic review of South Asia longtail macaque, Macaca fascicularis (Raffles, 1821). Fieldiana Zoology 1995, 81:1-206.

3. Kumar S, Hedges SB: TimeTree2: species divergence times on the iPhone. Bioinformatics 2011, 27(14):2023-2024.

4. Rhesus Macaque Genome Sequencing and Analysis Consortium: Evolutionary and biomedical insights from the rhesus macaque genome. Science 2007, 316:222-234.

5. Demands for Rehsus Monkeys in Biomedical Research: A Workshop Report. In: Workshop on Demands for Rhesus Monkeys in Biomedical Research 2002, 44:222-235.

6. Yan G, Zhang G, Fang X, Zhang Y, Li C, Ling F, Cooper DN, Li Q, Li Y, van Gool AJ, et al: Genome sequencing and comparison of two nonhuman primate animal models, the cynomolgus and Chinese rhesus macaques. Nat Biotechnol 2011, 29(11):1019-23.

7. Rowe N: The pictorial guide to the living primates. East Hampton, New York: Pogonias Press; 1996

8. Taylor K: Clinical veterinarian's perspective of non-human primate (NHP) use in drug safety studies. J Immunotoxicol 2010, 7:114-119.

9. Osada N, Hida M, Kusuda J, Tanuma R, Iseki K, Hirata M, Suto Y, Hirai M, Terao K, Suzuki Y, et al: Assignment of 118 novel cDNAs of cynomolgus monkey brain to human chromosomes. Gene 2001, 275:31-37.

10. Osada N, Hida M, Kusuda J, Tanuma R, Hirata M, Suto Y, Hirai M, Terao K, Sugano S, Hashimoto K: Cynomolgus monkey testicular cDNAs for discovery of novel human genes in the human genome sequence. BMC Genomics 2002, 3:36.

11. Osada N, Hirata M, Tanuma R, Kusuda J, Hida M, Suzuki Y, Sugano S, Gojobori T, Shen $\mathrm{CK}$, Wu Cl, et al: Substitution rate and structural divergence of 5'UTR evolution: comparative analysis between human and cynomolgus monkey cDNAs. Mol Biol Evol 2005, 22:1976-1982.

12. Osada N, Hashimoto K, Kameoka Y, Hirata M, Tanuma R, Uno Y, Inoue I, Hida M, Suzuki Y, Sugano S, et al: Large-scale analysis of Macaca fascicularis transcripts and inference of genetic divergence between $M$. fascicularis and M. mulatta. BMC Genomics 2008, 9:91.

13. Osada N, Hirata M, Tanuma R, Suzuki Y, Sugano S, Terao K, Kusuda J, Kameoka Y, Hashimoto K, Takahashi I: Collection of Macaca fascicularis CDNAs derived from bone marrow, kidney, liver, pancreas, spleen, and thymus. BMC Res Notes 2009, 2:199.

14. Magness CL, Fellin PC, Thomas MJ, Korth MJ, Agy MB, Proll SC, Fitzgibbon M, Scherer CA, Miner DG, Katze MG, et al: Analysis of the Macaca mulatta transcriptome and the sequence divergence between Macaca and human. Genome Biol 2005, 6:R60.

15. Uno $Y$, Suzuki $Y$, Wakaguri $H$, Sakamoto $Y$, Sano H, Osada N, Hashimoto $K$, Sugano S, Inoue I: Expressed sequence tags from cynomolgus monkey (Macaca fascicularis) liver: a systematic identification of drug-metabolizing enzymes. FEBS Lett 2008, 582:351-358.

16. Ebeling $M$, Küng $E$, See A, Broger $C$, Steiner $G$, Berrera $M$, Heckel $T$, Iniguez L Albert T, Schmucki R, et al: Genome-based analysis of the nonhuman primate Macaca fascicularis as a model for drug safety assessment. Genome Res 2011, 21(10):1746-1756.

17. Raabe BM, Lovaglio J, Grover GS, Brown SA, Boucher JF, Yuan Y, Civil JR, Gillhouse KA, Stubbs MN, Hoggatt AF, et al: Pharmacokinetics of cefovecin in cynomolgus macaques (Macaca fascicularis), olive baboons (Papio anubis), and rhesus macaques (Macaca mulatta). J Am Assoc Lab Anim Sci 2011, 50(3):389-395

18. Dembele L, Gego A, Zeeman AM, Franetich JF, Silvie O, Rametti A, Le Grand R, Dereuddre-Bosquet N, Sauerwein R, van Gemert GJ, et al: Towards an 
in vitro model of Plasmodium hypnozoites suitable for drug discovery. PLoS One 2011, 6(3):e18162.

19. Sánchez MG, Estrada-Camarena E, Bélanger N, Morissette M, Di Paolo T: Estradiol modulation of cortical, striatal and raphe nucleus 5-HT1A and 5-HT2A receptors of female hemiparkinsonian monkeys after long-term ovariectomy. Neuropharmacology 2011, 60(4):642-652.

20. Becker DP, Barta TE, Bedell LJ, Boehm TL, Bond BR, Carroll J, Carron CP, Decrescenzo GA, Easton AM, Freskos JN, et al: Orally active MMP-1 sparing a-tetrahydropyranyl and a-piperidinyl sulfone matrix metalloproteinase (MMP) inhibitors with efficacy in cancer, arthritis, and cardiovascular disease. J Med Chem 2010, 53(18):6653-6680.

21. Sasaki M, Kudo K, Honjo K, Hu JQ, Wang HB, Shintaku K: Prediction of infarct volume and neurologic outcome by using automated multiparametric perfusion-weighted magnetic resonance imaging in a primate model of permanent middle cerebral artery occlusion. J Cereb Blood Flow Metab 2011, 31(2):448-456.

22. Saiki H, Hayashi $T$, Takahashi $R$, Takahashi J: Objective and quantitative evaluation of motor function in a monkey model of Parkinson's disease. J Neurosci Methods 2010, 190(2):198-204

23. Arce F, Novick I, Mandelblat-Cerf Y, Israel Z, Ghez C, Vaadia E: Combined adaptiveness of specific motor cortical ensembles underlies learning. J Neurosci 2010, 30(15):5415-5425.

24. Burns SP, Xing D, Shelley MJ, Shapley RM: Searching for autocoherence in the cortical network with a time-frequency analysis of the local field potential. J Neurosci 2010, 30(11):4033-4047.

25. Dufrane D, Goebbels RM, Gianello P: Alginate macroencapsulation of pig islets allows correction of streptozotocin-induced diabetes in primates up to 6 months without immunosuppression. Transplantation 2010, 90 (10):1054-1062.

26. Shook BC, Rassnick S, Osborne MC, Davis S, Westover L, Boulet J, Hall D, Rupert KC, Heintzelman GR, Hansen $\mathrm{K}$, et al: In vivo characterization of a dual adenosine A2A/A1 receptor antagonist in animal models of Parkinson's disease. J Med Chem 2010, 53(22):8104-8115.

27. Hodgson RA, Bedard PJ, Varty GB, Kazdoba TM, Di Paolo T, Grzelak ME, Pond AJ, Hadjtahar A, Belanger N, Gregoire L, et al: Preladenant, a selective $\mathrm{A}(2 \mathrm{~A})$ receptor antagonist, is active in primate models of movement disorders. Exp Neurol 2010, 225(2):384-390.

28. Sasaki M, Kudo K, Honjo K, Hu JQ, Wang HB, Shintaku K: Prediction of infarct volume and neurologic outcome by using automated multiparametric perfusion-weighted magnetic resonance imaging in a primate model of permanent middle cerebral artery occlusion. J Cereb Blood Flow Metab 2011, 31(2):448-456.

29. Dimijian GG: Pathogens and parasites: strategies and challenges. Proc (Bayl. Univ. Med. Cent.) 2000, 13:19-29.

30. Raabe BM, Lovaglio J, Grover GS, Brown SA, Boucher JF, Yuan Y, Civil JR, Gillhouse KA, Stubbs MN, Hoggatt AF, et al: Pharmacokinetics of cefovecin in cynomolgus macaques (Macaca fascicularis), olive baboons (Papio anubis), and rhesus macaques (Macaca mulatta). J Am Assoc Lab Anim Sci 2011, 50(3):389-395.

31. Higashino A, Kageyama T, Kantha SS, Terao K: Detection of elevated antibody against calreticulin by ELISA in aged cynomolgus monkey plasma. Zoolog Sci 2011, 28(2):85-89.

32. Luquin MR, Manrique M, Guillén J, Arbizu J, Ordoñez C, Marcilla I: Enhanced GDNF expression in dopaminergic cells of monkeys grafted with carotid body cell aggregates. Brain Res 2011, 1375:120-127.

33. Dembele L, Gego A, Zeeman AM, Franetich JF, Silvie O, Rametti A, Le Grand $R$, Dereuddre-Bosquet N, Sauerwein $R$, van Gemert GJ, et al: Towards an in vitro model of Plasmodium hypnozoites suitable for drug discovery. PLoS One 2011, 6(3):e18162

34. Goff AJ, Chapman J, Foster C, Wlazlowski C, Shamblin J, Lin K, Kreiselmeier N, Mucker E, Paragas J, Lawler J, et al: A novel respiratory model of infection with monkeypox virus in cynomolgus macaques. J Virol 2011, 85(10):4898-4909.

35. Lemon $\mathrm{K}$, de Vries RD, Mesman AW, McQuaid S, van Amerongen G, Yüksel S, Ludlow M, Rennick LJ, Kuiken T, Rima BK, et al: Early target cells of measles virus after aerosol infection of non-human primates. PLOS Pathog 2011, 7(1):e1001263.

36. Feng M, Zhu H, Zhu Z, Wei J, Lu S, Li Q, Zhang N, Li G, Li F, Ma W, et al: Serial 18 F-FDG PET demonstrates benefit of human mesenchymal stem cells in treatment of intracerebral hematoma: a translational study in a primate model. J Nucl Med 2011, 52(1):90-97.
37. Igarashi Y, D'hoore W, Goebbels RM, Gianello P, Dufrane D: Beta-5 score to evaluate pig islet graft function in a primate pre-clinical model. Xenotransplantation 2010, 17(6):449-459.

38. Blauwblomme T, Piallat B, Fourcade A, David O, Chabardès S: Cortical stimulation of the epileptogenic zone for the treatment of focal motor seizures: an experimental study in the nonhuman primate. Neurosurgery 2011, 68(2):482-490.

39. Shook BC, Rassnick S, Osborne MC, Davis S, Westover L, Boulet J, Hall D, Rupert KC, Heintzelman GR, Hansen K, et al: In vivo characterization of a dual adenosine $A 2 \mathrm{~A} / \mathrm{A} 1$ receptor antagonist in animal models of Parkinson's disease. J Med Chem 2010, 53(22):8104-8115.

40. Warren R, Lockman H, Barnewall R, Krile R, Blanco OB, Vasconcelos D, Price J, House RV, Bolanowksi MA, Fellows P: Cynomolgus macaque model for pneumonic plague. Microb Pathog 2011, 50(1):12-22

41. Weissheimer KV, Herod SM, Cameron JL, Bethea $\mathrm{CL}$ : Interactions of corticotropin-releasing factor, urocortin and citalopram in a primate model of stress-induced amenorrhea. Neuroendocrinology 2010, 92(4):224-234.

42. Shahryarinejad A, Gardner TR, Cline JM, Levine WN, Bunting HA, Brodman MD Ascher-Walsh CJ, Scotti RJ, Vardy MD: Effect of hormone replacement and selective estrogen receptor modulators (SERMs) on the biomechanics and biochemistry of pelvic support ligaments in the cynomolgus monkey (Macaca fascicularis). Am J Obstet Gynecol 2010, 202(5):e1-9. 485.

43. Freeman WM, Salzberg AC, Gonzales SW, Grant KA, Vrana KE: Classification of alcohol abuse by plasma protein biomarkers. Biol Psychiatry 2010, 68(3):219-222

44. Kavanagh K, Brown KK, Berquist ML, Zhang L, Wagner JD: Fluid compartmental shifts with efficacious pioglitazone therapy in overweight monkeys: implications for peroxisome proliferator-activated receptor-gamma agonist use in prediabetes. Metabolism 2010, 59(6):914-920.

45. Tomkinson A, Tepper J, Morton M, Bowden A, Stevens L, Harris P, Lindell D, Fitch N, Gundel R, Getz EB: Inhaled vs subcutaneous effects of a dual IL-4/ IL-13 antagonist in a monkey model of asthma. Allergy 2010, 65(1):69-77.

46. Jerome C, Missbach M, Gamse R: Balicatib, a cathepsin K inhibitor, stimulates periosteal bone formation in monkeys. Osteoporos Int 2011, 22(12):3001-3011.

47. Alt FW, Bothwell AL, Knapp M, Siden E, Mather E, Koshland M, Baltimore D: Synthesis of secreted and membrane-bound immunoglobulin mu heavy chains is directed by mRNAs that differ at their 3 ' ends. Cell 1980, 20:293-301.

48. Early P, Rogers J, Davis M, Calame K, Bond M, Wall R, Hood L: Two mRNAs can be produced from a single immunoglobulin mu gene by alternative RNA processing pathways. Cell 1980, 20:313-319.

49. Rosenfeld MG, Lin CR, Amara SG, Stolarsky L, Roos BA, Ong ES, Evans RM: Calcitonin mRNA polymorphism: peptide switching associated with alternative RNA splicing events. Proc Natl Acad Sci U S A 1982, 79:1717-1721.

50. Lee C, Wang Q: Bioinformatics analysis of alternative splicing. Brief Bioinform 2005, 6:23-33.

51. Pan Q, Shai O, Lee LJ, Frey BJ, Blencowe BJ: Deep surveying of alternative splicing complexity in the human transcriptome by high-throughput sequencing. Nat Genet 2008, 40:1413-1415.

52. Landry JR, Mager DL, Wilhelm BT: Complex controls: the role of alternative promoters in mammalian genomes. Trends Genet 2003, 19:640-648.

53. Tazi J, Bakkour N, Stamm S: Alternative splicing and disease. Biochim Biophys Acta 2009, 1792(1):14-26.

54. Garcia-Blanco MA, Baraniak AP, Lasda EL: Alternative splicing in disease and therapy. Nat Biotechnol 2004, 22(5):535-546.

55. Orengo JP, Cooper TA: Alternative splicing in disease. Adv Exp Med Biol 2007, 23:212-223.

56. Nissim-Rafinia M, Kerem B: Splicing regulation as a potential genetic modifier. Trends Genet 2002, 18(3):123-127.

57. Buratti E, Baralle M, Baralle FE: Defective splicing, disease and therapy: searching for master checkpoints in exon definition. Nucleic Acids Res 2006, 34:3494-3510.

58. Faustino NA, Cooper TA: Pre-mRNA splicing and human disease. Genes Dev 2003, 17:419-437.

59. van de Lagemaat LN, Landry JR, Mager DL, Medstrand P: Transposable elements in mammals promote regulatory variation and diversification of genes with specialized functions. Trends Genet 2003, 19(10):530-536. 
60. Huh JW, Kim YH, Kim DS, Park SJ, Lee SR, Kim SH, Kim E, Kim SU, Kim MS, Kim HS, et al: Alu-derived old world monkeys exonization event and experimental validation of the LEPR gene. Mol Cells 2010, 30(3):201-207.

61. Zhou X, Ren L, Meng Q, Li Y, Yu Y, Yu J: The next-generation sequencing technology and application. Protein Cell 2010, 1(6):520-536.

62. Metzker ML: Sequencing technologies - the next generation. Nat Rev Genet 2010, 11(1):31-46.

63. Tsang WY, Spektor A, Luciano DJ, Indjeian VB, Chen Z, Salisbury JL, Sánchez I, Dynlacht BD: CP110 cooperates with two calcium-binding proteins to regulate cytokinesis and genome stability. Mol Biol Cell 2006, 17:3423-3434.

64. Zhang $\mathrm{XH}$, Chasin LA: Comparison of multiple vertebrate genomes reveals the birth and evolution of human exons. Proc Natl Acad Sci U S A 2006, 103(36):13427-13432.

65. Brett D, Hanke J, Lehmann G, Haase S, Delbrück S, Krueger S, Reich J, Bork P: EST comparison indicates $38 \%$ of human mRNAs contain possible alternative splice forms. FEBS Lett 2000, 474:83-86.

66. International Human Genome Sequencing Consortium: Initial sequencing and analysis of the human genome. Nature 2001, 409:860-921.

67. Calarco JA, Xing Y, Cáceres M, Calarco JP, Xiao X, Pan Q, Lee C, Preuss TM, Blencowe BJ: Global analysis of alternative splicing differences between humans and chimpanzees. Genes Dev 2007, 21(22):2963-2975.

68. Locke DP, Hillier LW, Warren WC, Worley KC, Nazareth LV, Muzny DM, Yang SP, Wang Z, Chinwalla AT, Minx P, et al: Comparative and demographic analysis of orang-utan genomes. Nature 2011, 469(7331):529-533.

69. Chimpanzee Sequencing and Analysis Consortium: Initial sequence of the chimpanzee genome and comparison with the human genome. Initial sequence of the chimpanzee genome and comparison with the human genome. Nature 2005, 437(7055):69-87.

70. Sverdlov ED: Retroviruses and primate evolution. Bioessays 2000, 22:161-171.

71. Speek M: Antisense promoter of human L1 retrotransposon drives transcription of adjacent cellular genes. Mol Cell Biol 2001, 21:1973-1985.

72. Lev-Maor G, Sorek R, Shomron N, Ast G: The birth of an alternatively spliced exon: 3' splice-site selection in Alu exons. Science 2003, 300:1288-1291.

73. Sela N, Mersch B, Gal-Mark N, Lev-Maor G, Hotz-Wagenblatt A, Ast G: Comparative analysis of transposed element insertion within human and mouse genomes reveals Alu's unique role in shaping the human transcriptome. Genome Biol 2007, 8:R127.

74. Sen SK, Han K, Wang J, Lee J, Wang H, Callinan PA, Dyer M, Cordaux R, Liang $P$, Batzer MA: Human genomic deletions mediated by recombination between Alu elements. Am J Hum Genet 2006, 79(1):41-53.

75. Han K, Lee J, Meyer TJ, Remedios P, Goodwin L, Batzer MA: L1 recombination-associated deletions generate human genomic variation. Proc Natl Acad Sci U S A 2008, 105(49):19366-19371.

doi:10.1186/1471-2164-13-163

Cite this article as: Huh et al:: Large-scale transcriptome sequencing and gene analyses in the crab-eating macaque (Macaca fascicularis) for biomedical research. BMC Genomics 2012 13:163.

\section{Submit your next manuscript to BioMed Central and take full advantage of:}

- Convenient online submission

- Thorough peer review

- No space constraints or color figure charges

- Immediate publication on acceptance

- Inclusion in PubMed, CAS, Scopus and Google Scholar

- Research which is freely available for redistribution 